\title{
Differential Immune Response against Recombinant Leishmania donovani Peroxidoxin 1 and Peroxidoxin 2 Proteins in BALB/c Mice
}

\author{
Nada S. Daifalla, ${ }^{1,2}$ Abebe Genetu Bayih, ${ }^{1}$ and Lashitew Gedamu ${ }^{1}$ \\ ${ }^{1}$ Department of Biological Sciences, University of Calgary, Room 374, 2500 University Drive NW, Calgary, AB, Canada T2N $1 N 4$ \\ ${ }^{2}$ The Forsyth Institute, Cambridge, MA 02142, USA \\ Correspondence should be addressed to Lashitew Gedamu; lgedamu@ucalgary.ca
}

Received 28 January 2015; Accepted 25 April 2015

Academic Editor: Diana Boraschi

Copyright (C) 2015 Nada S. Daifalla et al. This is an open access article distributed under the Creative Commons Attribution License, which permits unrestricted use, distribution, and reproduction in any medium, provided the original work is properly cited.

\begin{abstract}
We assessed the immune response against recombinant proteins of two related, albeit functionally different, peroxidoxins from Leishmania donovani: peroxidoxin 1 (LdPxn1) and peroxidoxin 2 (LdPxn2) in BALB/c mice. We also evaluated the effect of coadministration of TLR agonists (CpG ODN and GLA-SE) on the antigen-specific immune response. Immunization with recombinant LdPxn1 alone induced a predominantly Th2 type immune response that is associated with the production of high level of IgG1 and no IgG2a isotype while rLdPxn2 resulted in a mixed Th1/Th2 response characterized by the production of antigenspecific IgG2a in addition to IgG1 isotype. Antigen-stimulated spleen cells from mice that were immunized with rLdPxn1 produced low level of IL-10 and IL-4 and no IFN- $\gamma$, whereas cells from mice immunized with rLdPxn2 secreted high level of IFN- $\gamma$, low IL-4, and no IL-10. Coadministration of CpG ODN or GLA-SE with rLdPxn1 skewed the immune response towards a Th 1 type as indicated by robust production of IgG2a isotype. Furthermore, the presence of TLR agonists together with rLdPxn1 antigen enhanced the production of IFN- $\gamma$ and to a lesser extent of IL-10. TLR agonists also enhanced a more polarized Th 1 type immune response against rLdPxn2.
\end{abstract}

\section{Introduction}

Infection by parasites of the genus Leishmania results in a chronic disease known as leishmaniasis. It is transmitted when an infected female phlebotomine sandfly injects the metacyclic promastigotes into the host during a blood meal. The flagellated promastigotes are taken by macrophages where they transform into aflagellated amastigotes that multiply and disseminate the infection [1]. The outcome of Leishmania infection depends on the species of Leishmania as well as the host immune response. Clinical manifestation of leishmaniasis ranges from self-healing cutaneous form to fatal visceral disease [2]. The disease is prevalent worldwide infecting millions of people in more than 90 countries in the tropics, subtropics, and southern Europe (Center for Disease Control and Prevention, http://www.cdc.gov/parasites/leishmaniasis/). About 1.3 million new cases and about 30000 deaths are recorded each year with the majority of these cases occurring in poor regions of the world (World Health Organization, http://www.who.int/mediacentre/factsheets/fs375/en/, [3]) where the afflicted populations have low accessibility to health care. Chemotherapy is available but its usefulness is compromised by toxicity of some drugs and drug resistance by the parasite [4]. In addition, the emergence of Leishmania/HIV coinfection compounded the problem. Concomitant infection with HIV increases the cases of active VL in otherwise asymptomatic individuals by 100 to 1000 times and it increases the likelihood of drug toxicity as well as relapse of the disease [5].

Experimental studies have shown that protection against leishmaniasis is mediated by Thelper 1 (Th1) type $\mathrm{CD} 4^{+}$cells that produce a high level of interferon gamma (IFN- $\gamma$ ) and tumor necrosis factor alpha (TNF- $\alpha$ ) whereas progression of the disease is associated with $\mathrm{Th} 2$ type $\mathrm{CD} 4^{+}$cells which produce IL-4, IL-5, IL-10, and IL-13 [6-9]. The Th1 and Th2 cells 


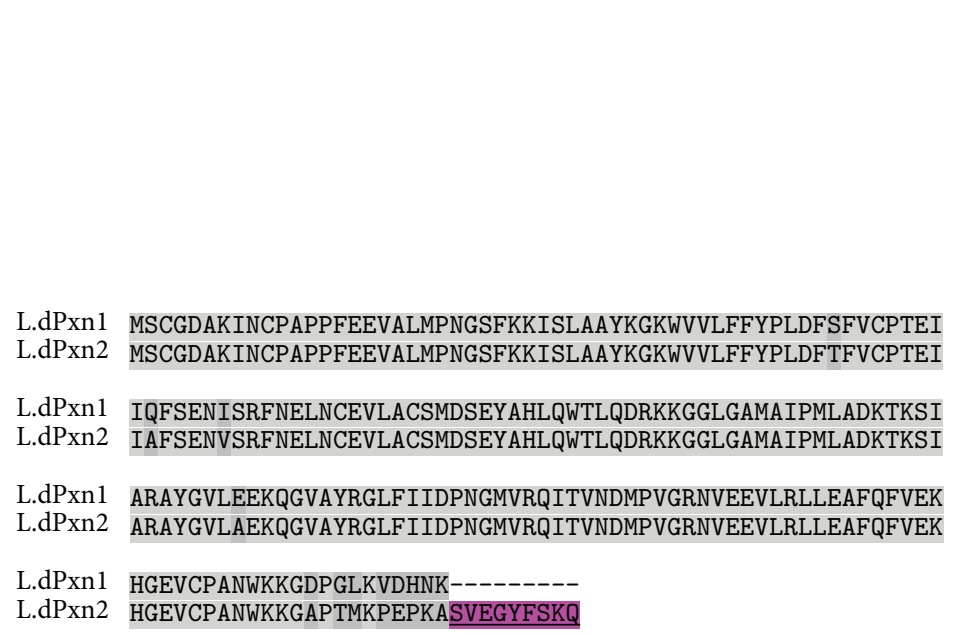

(a)

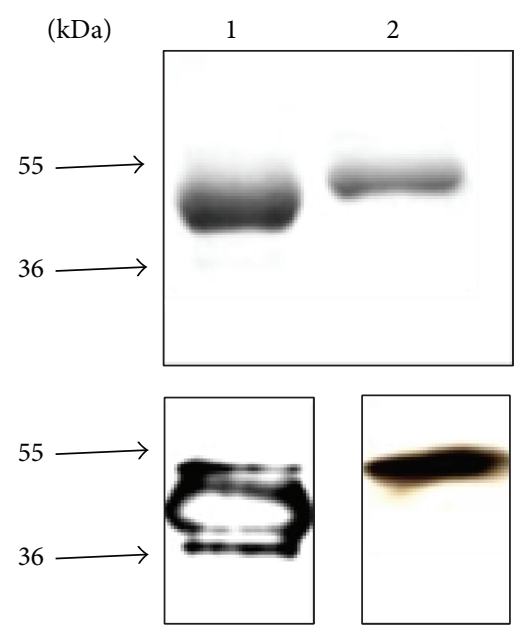

(b)

Figure 1: (a) Sequence comparison of Leishmania donovani Pxn1 and Pxn2. Alignment of amino acid sequence depicts the high homology between LdPxn1 and LdPxn2. Highlighted areas show positions of mismatch. LdPxn2 possesses extra 9 amino acids at the carboxy terminus (underlined) that are missing from LdPxn1. (b) SDS-PAGE and western blot of rLdPxn1 and rLdPxn2 proteins. One microgram per lane of rLdPxn1 (lane 1) and rLdPxn2 (lane 2) was separated on a 12\% SDS-PAGE and stained with Coomassie blue, top. The separated samples were transferred to Hybond-P membrane and were probed with pooled sera from mice immunized with the respective recombinant protein, bottom. Molecular weight in $\mathrm{kDa}$ is shown on the left.

have differential capabilities in stimulating B cells to secrete different antibody isotypes where Th1 type cells elicit IgG2a antibody production and Th2 type cells induce IgG1 antibody secretion [10]. This differential effect is brought about by the regulatory effect of cytokines on the immunoglobulin isotype switching. In vitro studies have shown that IL-4 and IFN$\gamma$ stimulate the production of IgG1 and IgG2a, respectively $[11,12]$.

Leishmania parasites are highly successful in parasitizing macrophage cells which are otherwise hostile to pathogens. Generally, uptake of pathogenic organisms by macrophages results in oxidative burst which is associated with the production of reactive oxygen species (ROS) such as superoxide radical $\left(\mathrm{O}_{2}{ }^{-\bullet}\right)$, hydrogen peroxide $\left(\mathrm{H}_{2} \mathrm{O}_{2}\right)$, and hydroxyl anion $\left(\mathrm{OH}^{-}\right)$and reactive nitrogen species (RNS) including nitric oxide (NO). These reactive species are highly destructive to the infecting pathogen and they can interact with each other forming more potent oxidants such as peroxynitrite $\left(\mathrm{ONOO}^{-}\right)[13]$.

One of the evasive mechanisms used by Leishmania parasites to bypass the microbicidal effect of free radicals produced by macrophages is the expression of antioxidant enzymes known as peroxidoxins. These enzymes are conserved and highly abundant proteins in almost all living organisms which suggest essential function in oxidative homeostasis. It has been shown that peroxidoxins from different organisms including Leishmania are important in the protection of these organisms against oxidative stress [1416]. We isolated and characterized three peroxidoxins as part of a multigene family from $L$. donovani complex: Pxn1, Pxn2, and Pxn3 [14, 17]. Both Pxn1 and Pxn2 are cytosolic whereas $\mathrm{Pxn} 3$ is predicted to be glycosomal. A fourth mitochondrial peroxidoxin, Pxn4, has also been identified in $L$. donovani [18]. In addition to the common localization of Pxn1 and Pxn2 in the cytoplasm, the two proteins have $89.4 \%$ homology. The difference between these two proteins is brought about by an extra 9 amino acids at the carboxy terminus of Pxn2 plus few nucleotide mismatches along the entire sequence $[14,17]$ (Figure 1(a)). Despite the high similarity between LdPxnl and LdPxn2 at the amino acid level, there are striking differences between the proteins encoded by the two genes. Unlike LdPxnl, which is upregulated during the amastigote stage, LdPxn2 is expressed at high levels during the promastigote stage and the expression declines towards the amastigote stage. In addition, while recombinant LdPxnl protein has been shown to detoxify various free radicals including ROS and RNS, LdPxn2 can only detoxify $\mathrm{H}_{2} \mathrm{O}_{2}$ [14].

In this study, we assessed the immune responses against LdPxn1 and LdPxn2 as recombinant GST-fusion proteins in $\mathrm{BALB} / \mathrm{c}$ mice to test if the differences observed in gene expression and functionality between these two antigens are paralleled by different immune response profile. In addition, we evaluated the immune response against these proteins in the presence of two Th1 adjuvants: bacterial $\mathrm{CpG}$ oligodeoxynucleotide (CpG ODN) and glucopyranosyl lipid $\mathrm{A}$ in a stable emulsion (GLA-SE), which are Toll-like receptor 9 (TLR-9) and TLR-4 agonists, respectively. Our results indicate that mice immunization with LdPxnl induces a predominant Th2 type response, whereas immunization with LdPxn2 stimulates a mixed Th1/Th2 response. Our data also show that repeated injections with coadministration of Th1adjuvants enhanced the immune response against LdPxn1 and LdPxn2 which is more biased towards Th1 type.

\section{Materials and Methods}

2.1. Mice. Female BALB/c mice (4-6 weeks old) were purchased from Charles River Laboratories (QC, Canada) and 


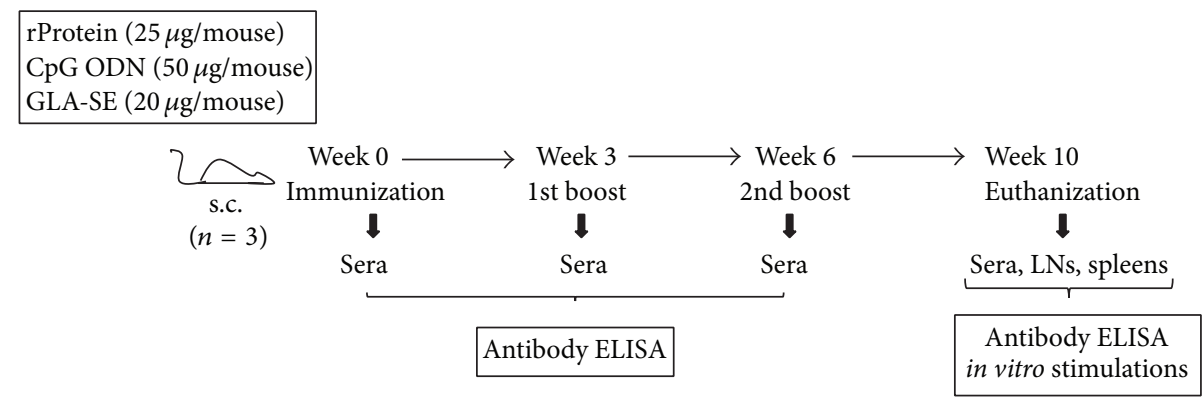

FIGURE 2: Schematic representation of the immunization protocol. Six- to 8-week-old female BALB/c mice were immunized s.c. in the hind foot pad with recombinant LdPxn1 or LdPxn2 protein ( $25 \mathrm{ug} / \mathrm{mouse}$ ) with or without CpG ODN (50 ug/mouse) or GLA-SE (20 ug/mouse) adjuvants. Mice were boosted twice in 3 weeks interval. Sera were collected at each time of injection. Four weeks after the last boost mice were euthanized and sera, lymph nodes, and spleens were collected. Samples were used for antibody and cytokine analysis using standard protocols.

were housed in a specific pathogen-free facility at the University of Calgary and provided water and food ad libitum. Mice were acclimatized for one week and randomly distributed into experimental groups and controls. Animal protocols were approved by the Life and Environmental Sciences Animal Care Committee (LESACC) of the University of Calgary, Alberta, Canada.

\subsection{Cloning, Expression, and Purification of Recombinant} LdPxn1 and LdPxn2 in E. coli. Cloning of LdPxn1 and LdPxn2 as GST-fusion proteins was performed by using the prokaryotic expression vector, pGEX-2T (Amersham Pharmacia Biotech) following the procedure described previously [17]. Briefly, the coding regions of LdPxnl and LdPxn2 were amplified by PCR using specific primers. The amplified fragments were then cloned into pGEX-2T vector. To express the recombinant proteins, transformed E. coli BL21 (DE3) cells were grown in a $37^{\circ} \mathrm{C}$ shaker overnight in Luria-Bertani (LB) broth in the presence of $100 \mu \mathrm{g} / \mathrm{mL}$ ampicillin. The cultures were induced with $0.2 \mathrm{mM}$ isopropyl beta-D-thiogalactoside (IPTG) and continued to grow for 3-6 hours. Fusion proteins, GST-LdPxn1 and GST-LdPxn2, were harvested by sonication and passing over a glutathioneagarose resin column (Sigma) as described by Smith and Johnson [19]. Endotoxins were removed using Detoxi-Gel Affinity Pak prepacked columns following the manufacture's instruction (Pierce Biotechnology, USA). Endotoxin level of protein samples was measured at the Infectious Disease Research Institute (Seattle, USA) using Limulus Amebocyte Lysate (LAL) assay. Samples of endotoxin levels $<10 \mathrm{EU} / \mathrm{mg}$ protein were used.

2.3. Immunization. Immunization protocol is schematically represented in Figure 2. Mice were randomly divided into groups of three and were immunized subcutaneously (s.c.) with recombinant LdPxn1, rLdPxn1 plus CpG ODN, rLdPxn1 plus GLA-SE, rLdPxn2, rLdPxn2 plus CpG ODN, and rLdPxn2 plus GLA-SE. Recombinant proteins, CpG ODN 1826 (Coley Pharmaceutical Group, Canada) and GLA-SE (Infectious Disease Research Institute, Seattle, USA), were given at 25,50 , and $20 \mu \mathrm{g} /$ mouse, respectively. Two booster injections were given in three-week interval. Sera were isolated from blood (collected every three weeks starting from the time of first immunization (week 0) until the time of euthanization) and stored at $-20^{\circ} \mathrm{C}$. Mice were euthanized four weeks after the last boost, and lymph node and spleens were aseptically harvested and processed for the isolation of single cell suspensions. The isolated lymph node and spleen cells were used for in vitro antigen stimulation experiments.

2.4. Western Blotting. For western blotting, $1 \mu \mathrm{g}$ of each of the recombinant proteins was separated by SDS-PAGE and transferred to Hybond-P membrane (GE Healthcare, QC, Canada). The membrane was blocked with $5 \%$ skim milk dissolved in phosphate buffered saline (PBS) containing $0.05 \%$ tween-20 (PBS-T) for $2 \mathrm{hr}$ at room temperature. Then, it was incubated overnight at $4^{\circ} \mathrm{C}$ with mice serum that was collected four weeks after the last immunization with the respective antigen. After washing three times with PBS-T, the membrane was incubated with a horseradish peroxidaseconjugated anti-mouse IgG (GE Healthcare, QC, Canada) for $45 \mathrm{~min}$ at room temperature (RT) followed by three washing steps. Immunoreactivity was detected by chemiluminescence using ECL reagents following the manufacturer's instructions (GE Healthcare, QC, Canada).

2.5. Antibody Measurement. The presence of antibody specific to LdPxn1 and LdPxn2 in serum samples was determined by enzyme-linked immunosorbent assay (ELISA). Briefly, 96well microtiter plates (Sarstedt, USA) were coated overnight at $4^{\circ} \mathrm{C}$ with $1 \mu \mathrm{g} / \mathrm{mL}$ recombinant protein in bicarbonate buffer, $\mathrm{pH}$ 9.6. The plates were blocked with $5 \%(\mathrm{w} / \mathrm{v})$ skim milk in PBS-T for $1 \mathrm{hr}$ at RT. After three washes with PBS-T, $100 \mu \mathrm{l} /$ well of sera diluted 1:100 in blocking buffer was added to the plates and incubated for $1 \mathrm{hr}$ at RT. After washing, $100 \mu \mathrm{l} /$ well of biotinylated goat anti-mouse IgG1 or IgG2a antibody was added to the wells and incubated for $1 \mathrm{hr}$ at RT followed by $1 \mathrm{hr}$ incubation with streptavidin-HRP. The reaction was then developed by adding $100 \mu \mathrm{l} /$ well TMB $\left(3,3^{\prime}, 5,5^{\prime}\right.$-tetramethylbenzidine) substrate (BD Biosciences, ON, Canada). After the reaction was stopped by adding 


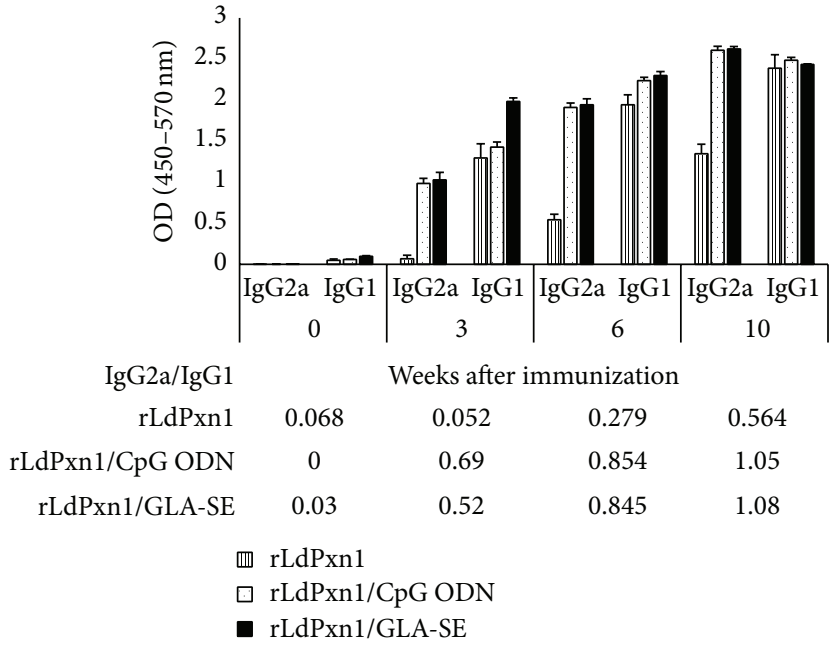

(a)

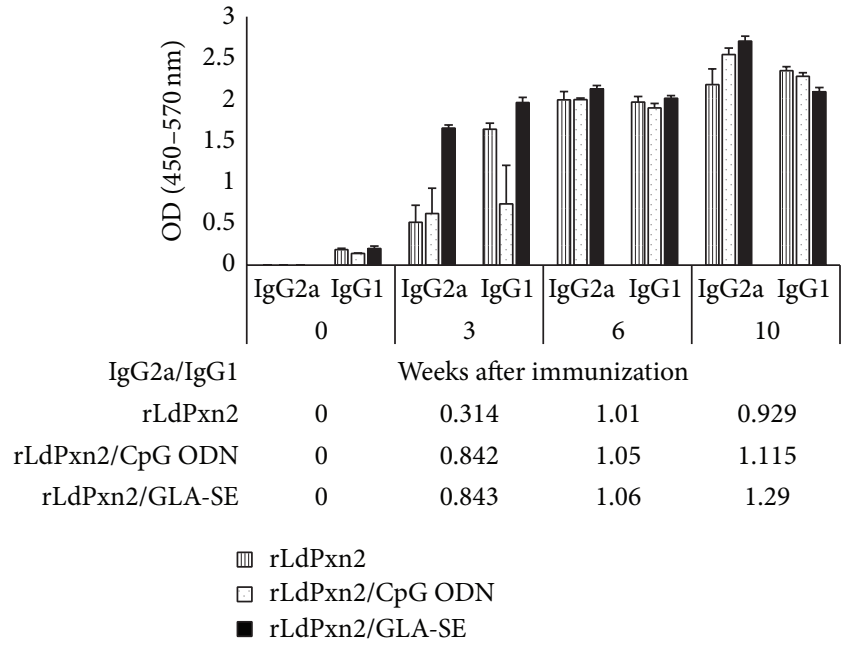

(b)

Figure 3: Anti-LdPxn1 and -LdPxn2 antibodies in immunized mice. Mice were immunized subcutaneously with recombinant LdPxn1 or LdPxn2 proteins with or without CpG ODN or GLA-SE. Mice were boosted twice in 3-week intervals. The levels of IgG1 and IgG2a isotypes were measured on sera collected at different time points using ELISA. Data are presented as the mean OD \pm S.E.M. of IgG1 and IgG2a of sera from mice immunized with rLdPxn1 (a) and rLdPxn2 (b). The IgG2a/IgG1 ratios are shown in tables below each figure.

$50 \mu \mathrm{l} /$ well $1 \mathrm{~N} \mathrm{H}_{2} \mathrm{SO}_{4}$, the plates were read at $450 \mathrm{~nm}$ in a microplate reader (Molecular Devices, USA).

\subsection{In Vitro Antigen Stimulation and Cytokine Measurement.} Mice were euthanized 4 weeks after the last immunization and lymph node and spleen cells were isolated as described previously [20]. Cells from lymph nodes of mice from the same group were pooled before in vitro stimulation. For stimulation assays, cells from individual spleens or from pooled lymph nodes were dispensed at $2 \times 10^{5}$ cell/100 $\mu \mathrm{L}$ media/well in 96-well flat bottomed tissue culture plates (Sarstedt, USA) and incubated with 2 or $10 \mu \mathrm{g} / \mathrm{mL}$ of recombinant protein in complete medium (RPMI-1640 supplemented with 10\% FBS, $2 \mathrm{mM}$ L-glutamine, $25 \mathrm{mM}$ HEPES, penicillin $(100 \mathrm{U} / \mathrm{ml})$ plus streptomycin $(100 \mathrm{lg} / \mathrm{mL})$, and $50 \mu \mathrm{M} \beta$ Mercaptoethanol) for $72 \mathrm{hr}$ at $37^{\circ} \mathrm{C}$ in a humidified incubator with $5 \% \mathrm{CO}_{2}$. Cells were also incubated with $5 \mu \mathrm{g} / \mathrm{ml}$ concanavalin A (ConA) or with medium alone as positive and negative control, respectively. Culture supernatants were collected and cytokine production was measured using cytokine ELISA kits as per the manufacturer's instructions (BD Bioscience, ON, Canada) as described previously [20]. The amount of IFN- $\gamma$ and IL-10 produced by lymph node or spleen cells was expressed as $\mathrm{ng} / \mathrm{ml}$. In addition, the production of IL-4 was measured in spleen cells and was expressed as $\mathrm{pg} / \mathrm{mL}$.

2.7. Statistics. Data are expressed as the mean \pm standard error of the mean (S.E.M.). Statistical analysis was performed using Student's $t$-test. $P$ value of less than 0.05 was considered statistically significant.

\section{Results}

3.1. Recognition of LdPxn1 and LdPxn2 by Immune Sera. To demonstrate the immunoreactivity of recombinant LdPxn1 and LdPxn2 in BALB/c mice, we tested the interaction between sera collected from immunized mice and the respective recombinant protein by western blot analysis. As depicted in Figure 1(b), mice immune sera bound to the respective recombinant protein immobilized onto the membranes as indicated by the prominent bands of the expected molecular size of the GST-fused proteins. This indicates that both proteins are immunogenic in BALB/c mice.

\subsection{Comparative Analysis of Humoral Immune Response to} Recombinant LdPxn1 and LdPxn2 Proteins. To analyze the isotype profile of antibody response in mice immunized with rLdPxn1 or rLdPxn2, we measured antigen specific IgG1 and IgG2a isotypes in sera collected at different time points after immunization. In addition, we calculated the ratio of IgG2a to IgG1 as a surrogate marker for Thl type immune response.

As shown in Figure 3(a), immunization of mice with rLdPxn1 by itself stimulated a high level of IgG1 isotype and barely detectable amount of IgG2a at 3 weeks after the first immunization. The amount of specific IgG2a stimulated in this group increased upon booster immunization; however it remains significantly lower than the amount of $\operatorname{IgG1}(P<$ 0.05). Concomitant injection of CpG ODN or GLA-SE with rLdPxn1 triggered a high level of IgG1 and more importantly a high level of IgG2a as well (Figure 3(a)). Similar to immunization with rLdPxn1 alone, the production of anti-rLdPxn1 antibodies in mice immunized with rLdPxn1 plus adjuvants was augmented by booster immunization (Figure 3(a)). The augmentation effect of booster injections together with 


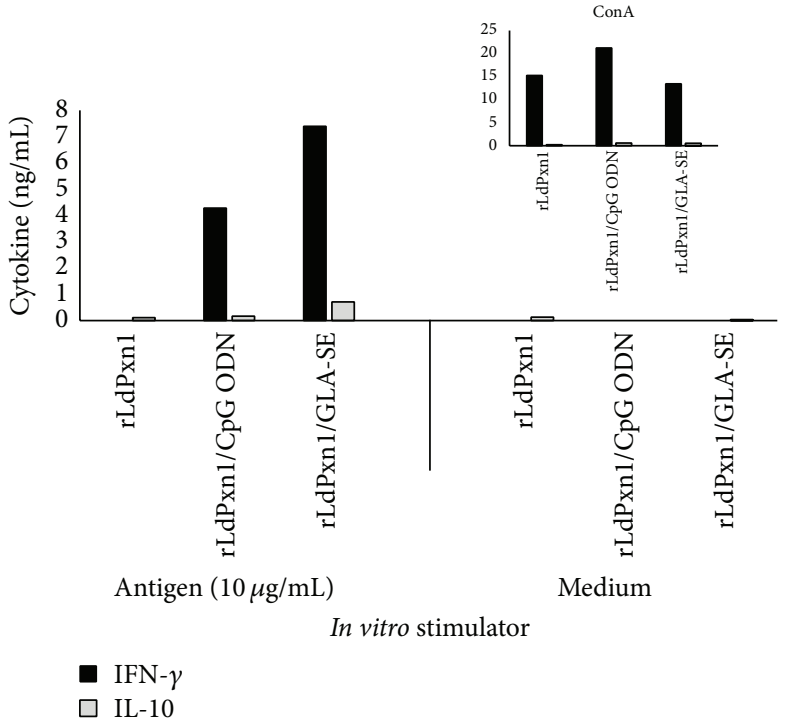

(a)

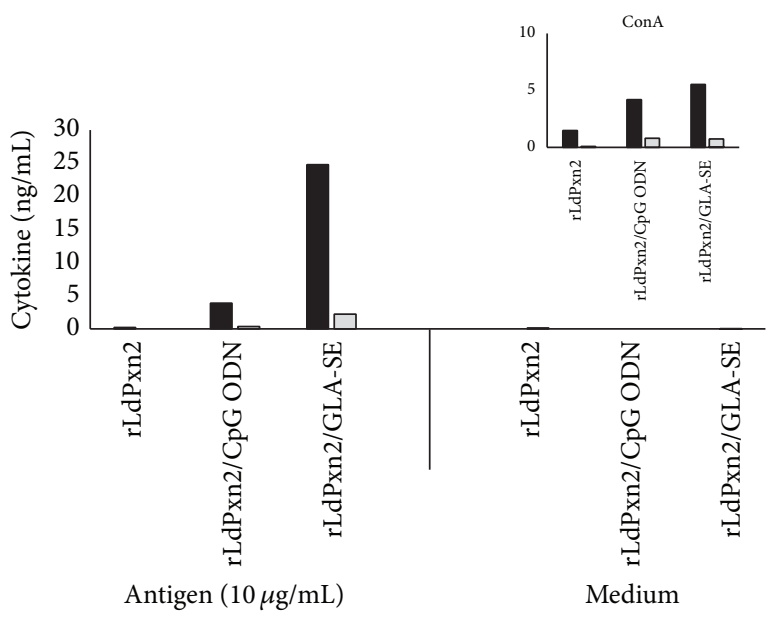

In vitro stimulator

- IFN- $\gamma$

IL-10

Figure 4: Cytokine responses in lymph node cells of rLdPxn1 and rLdPxn2 immunized mice. Mice were immunized subcutaneously three times at three-week intervals with rLdPxn1 or rLdPxn2 with or without CpG ODN or GLA-SE. Four weeks after the last immunization, cells from pooled lymph nodes were stimulated in vitro with the respective antigen $(10 \mu \mathrm{g} / \mathrm{mL})$ or ConA $(5 \mu \mathrm{g} / \mathrm{mL})$. The release of IFN- $\gamma$ and IL-10 in mice immunized with rLdPxn1 or rLdPxn2 was measured in supernatants after $72 \mathrm{hr}$ of in vitro stimulation at $37^{\circ} \mathrm{C}$. Results are presented as the amount of IFN- $\gamma(\mathrm{ng} / \mathrm{mL})$ and IL-10 (ng/mL) for rLdPxn1 (a) and rLdPxn2 (b).

the presence of TLR agonists in the immunization protocol resulted in the induction of IgG2a level as high as IgG1 four weeks after the last boost.

In contrast to rLdPxn1, rLdPxn2 alone was able to induce specific IgG2a production, in addition to IgG1 isotype, as early as 3 weeks after the first immunization (Figure 3(b)). The level of both isotypes was enhanced by booster immunizations in this group and the level of IgG2a was comparable to the level of IgG1 after the second injection, that is, the first boost (Figure 3(b)). Coadministration of $\mathrm{CpG} O \mathrm{ODN}$ or GLA-SE adjuvants with rLdPxn2 resulted in the production of high and comparable levels of both IgG2a and IgG1 isotypes as early as 3 weeks after the first boost (week 6) (Figure 3(b)). Coadministration of CpG ODN with rLdpxn2 induced lower antibody response as compared to GLA-SE after the first injection. However, the level of both isotypes in all groups receiving rLdPxn2 was comparable after the second booster immunization. At this time point, the level of IgG2a was slightly less or slightly more than IgG1 in the group immunized with rLdPxn2 alone or rLdPxn2 plus adjuvant, respectively.

These results indicate that, in $\mathrm{BALB} / \mathrm{c}$ mice, priming with recombinant LdPxn1 induces a predominantly Th2 response (IgG2a/IgG1 ratio of 0.052 ) whereas priming with recombinant LdPxn2 stimulates a mixed Th1/Th2 response (IgG2a/IgG1 ratio of 0.314 ). The data also reveal that, four weeks after the second boost, the ratio of $\operatorname{IgG} 2 \mathrm{a} / \mathrm{IgG} 1$ increased to 0.564 and 0.929 for rLdPxn1 and rLdPxn2, respectively. These results suggest that booster immunization can enhance the immune response against rLdPxnl and rLdPxn2. Our findings also show that CpG ODN and GLA-SE adjuvants have the capacity to skew the immune response against rLdPxn1 and rLdPxn2 toward a more Th1 type (IgG2a/IgG1 ratio > 1.0 after the last boost).

3.3. Antigen Specific Cellular Immune Response. To understand the type of cell-mediated immune response (CMI) against rLdPxn1 and rLdPxn2, we measured the level of IFN- $\gamma$ and IL-10 in antigen-stimulated lymph node cells and the level of IFN- $\gamma$, IL-10, and IL- 4 in the spleen cells of immunized mice. Lymph node cells from mice in each group were pooled and stimulated in vitro with $10 \mu \mathrm{g} / \mathrm{mL}$ of recombinant proteins whereas spleen cells from individual mouse were stimulated with 2 or $10 \mu \mathrm{g} / \mathrm{mL}$ of recombinant proteins. No stimulation or stimulation with $5 \mu \mathrm{g} / \mathrm{mL}$ Con A was added as negative and positive controls, respectively. Culture supernatants were collected $72 \mathrm{hr}$ later and the amount of cytokines was determined by ELISA.

There was no spontaneous release of cytokines by unstimulated lymph node cells in any of the groups (Figures 4(a) and 4(b)). No detectable cytokine was released by lymph node cells from mice immunized with the recombinant proteins alone (Figures 4(a) and 4(b)). However, immunization of mice with the recombinant proteins in the presence of TLR agonists resulted in the production of a high level of IFN$\gamma$ and a low level of IL-10 (Figures 4(a) and 4(b)). While coadministration of $\mathrm{CpG}$ ODN triggered the production of similar amounts of IFN- $\gamma$ in mice immunized with rLdPxn1 or rLdPxn2, GLA-SE stimulated the release of more IFN$\gamma$ in the group receiving $\operatorname{rLdPxn} 2$. The amount of IFN$\gamma$ produced by lymph node cells of mice from this group 
TABLE 1: The ratio of IFN- $\gamma /$ IL-10 in lymph node cells of immunized mice.

\begin{tabular}{lccccccccc}
\hline & \multicolumn{3}{c}{ Antigen $(10 \mu \mathrm{g} / \mathrm{mL})$} & \multicolumn{3}{c}{ Medium } & \multicolumn{2}{c}{ ConA } \\
& Antigen & +CpG ODN & +GLA-SE & Antigen & +CpG ODN & +GLA-SE & Antigen & +CpG ODN & +GLA-SE \\
\hline rLdPxn1 & 0 & 24.74 & 10.42 & 0 & 0 & 0 & 75.35 & 36.59 & 24.6 \\
rLdPxn2 & 0 & 10.83 & 11.11 & 0 & 0 & 0 & 19.42 & 5.19 & 7.38 \\
\hline
\end{tabular}

(Figure 4(b)) was more than 3-fold higher than the level produced by lymph node cells from mice immunized with rLdPxn1 alone (Figure 4(a)). Interestingly, CpG ODN and GLA-SE triggered the production of lower amount of IL-10 in mice receiving rLdPxn1 as compared to rLdPxn2 (Figures 4(a) and 4(b)). Lymph node cells stimulated with ConA mitogen produced comparable levels of cytokines (Figures 4(a) and $4(\mathrm{~b})$ in sets) with the exception of the group immunized with rLdPxn2 alone which produces lower level of cytokines (Figure 4(b) in set). The results of cytokine analyses in lymph node cells demonstrate that rLdPxn1 and rLdPxn2 can stimulate lymph node cells of immunized mice to produce cytokines only in the presence of adjuvants. The results also show that CpG ODN and GLA-SE adjuvants favor a Th1 type response against the two antigens as indicated by the high IFN- $\gamma /$ IL-10 ratios (Table 1). Moreover, the results show that while coadministration of GLA-SE exerts comparable effect on both antigens as indicated by comparable ratios of IFN- $\gamma /$ IL-10 (Table 1), CpG ODN induces stronger Th1 in mice receiving $r L d P x n 1$ as compared to $\operatorname{rLdPxn} 2$ (IFN- $\gamma / \mathrm{IL}$ 10 ratio of 24.74 and 10.83 for rLdPxn1 and rLdPxn2, resp.) (Table 1).

Production of IFN- $\gamma$, IL-10, and IL- 4 by spleen cells of immunized mice is depicted in Figure 5.

Spleen cells from mice immunized with rLdPxn1 by itself did not produce any detectable level of IFN- $\gamma$ and very low level of IL-10 upon in vitro stimulation with 2 or $10 \mu \mathrm{g} / \mathrm{ml}$ rLdPxn1 (Figure 5(a)). In contrast, immunization with rLdPxn2 alone was able to stimulate mice spleen cells to produce considerable amount of IFN- $\gamma$ but low IL-10 in in vitro recall experiments with 2 and $10 \mathrm{ug} / \mathrm{ml} \mathrm{rLdPxn} 2$ (Figure 5(b)). Both rLdPxn1 and rLdPxn2 stimulated the production of a low level of IL-4 in spleen cells of immunized mice when stimulated in vitro with $2 \mu \mathrm{g} / \mathrm{mL}$ of the respective protein. However, IL-4 production was only detected in spleen cells from the group receiving $\mathrm{rLdPxn} 2$ upon stimulation with $10 \mu \mathrm{g} / \mathrm{mL}$ (Figure 5(c)).

Administration of rLdPxnl in the presence of CpG ODN results in the production of a low level of IFN- $\gamma$ and almost no IL-10 when the spleen cells were stimulated with $2 \mu \mathrm{g} / \mathrm{ml}$ of the antigen (Figure 5(a)). At this concentration, stimulated spleen cells from mice receiving rLdPxn1 plus GLA-SE produced low but comparable levels of IFN- $\gamma$ and IL-10. The level of IFN- $\gamma$ and IL-10 produced by spleen cells from mice that received $\mathrm{rLdPxn} 1$ plus the adjuvants was dose-dependent with the production of higher levels of each cytokine upon stimulation with $10 \mu \mathrm{g} / \mathrm{mL}$ of the recombinant protein (Figure 5(a)).

In vitro stimulation of spleen cells from mice immunized with rLdPxn2 plus CpG ODN or GLA-SE with 2 and $10 \mu \mathrm{g} / \mathrm{ml}$ produced a high level of IFN- $\gamma$ and a low IL-10 (Figure 4(b)).
Interestingly, as shown in Figure 5(c), the presence of the TLR agonists in the immunization protocol induced the production of a higher level of IL- 4 by the spleen cells from mice immunized with $\operatorname{rLdPxn} 2$ as compared to low or none from those immunized with rLdPxn1. Spontaneous release of a low level of IL-10 by spleen cells from mice receiving rLdPxn1 by itself or with CpG ODN was observed (Figure 5(a)) as well as a low level of IL- 4 by spleen cells from mice immunized with rLdPxn 2 alone or plus CpG ODN (Figure 5(c)).

These results show that rLdPxn1 alone stimulates a weak cell-mediated immunity in the spleens of immunized mice as indicated by the low level or absence of detectable cytokines in in vitro recall experiments. Administration of rLdPxn1 in the presence of CpG ODN or GLA-SE increased the immune response with higher IFN- $\gamma / \mathrm{IL}-10$ ratio (3.78 and 2.03 for rLdPxn1-CPG ODN and rLdPxn1 GLA-SE, resp.) (Table 2) and low IL-4 (Figure 5(c)). On the other hand, immunization of mice with rLdPxn2, in the presence or absence of adjuvants, results in a mixed Th1/Th2 type response in spleen cells of immunized mice associated with high IFN- $\gamma /$ IL-10 ratio $(12.82,10.88$, and 7.55 for rLdPxn2, rLdPxn 2 CpG ODN, and rLdPxn2 GLA-SE, resp.) (Table 2) and high level of IL-4 (Figure 5(c)). This observation indicates that, independently of the adjuvant use, rLdPxn2 is capable of inducing a mixed Th1/Th2 response biased toward a Th1 type.

\section{Discussion}

In this study, we report differential immune responses against two cytosolic Leishmania donovani peroxidoxins: LdPxnl and LdPxn2. These two antioxidants are highly homologous, yet they are differentially expressed. The expression of LdPxn1 is upregulated during the mammalian amastigote stage whereas LdPxn2 is highly abundant in the promastigote stage [17]. In addition, LdPxn1 and LdPxn2 are functionally different; LdPxnl has been found to detoxify a wide range of reactive species (ROS and RNS) while LdPxn2 can only neutralize $\mathrm{H}_{2} \mathrm{O}_{2}$ [14]. The main focus of this work was to examine and compare the humoral and cellular immune responses against recombinant LdPxn1 and LdPxn2 GST-fusion proteins in $\mathrm{BALB} / \mathrm{c}$ mice and to investigate the potential of two TLR agonists as adjuvants that can be used with these recombinant proteins.

Our findings show that recombinant LdPxn1 protein induces a predominant Th2 type immune response in mice, whereas rLdPxn2 stimulates a mixed Th1/Th2 response biased toward a Thl type. Our data also demonstrate that coadministration of CpG ODN and GLA-SE favors the stimulation of a polarized Th1 type response with increased ratios of IgG2a/IgG1 and IFN- $\gamma / \mathrm{IL}-10$. This finding is not 


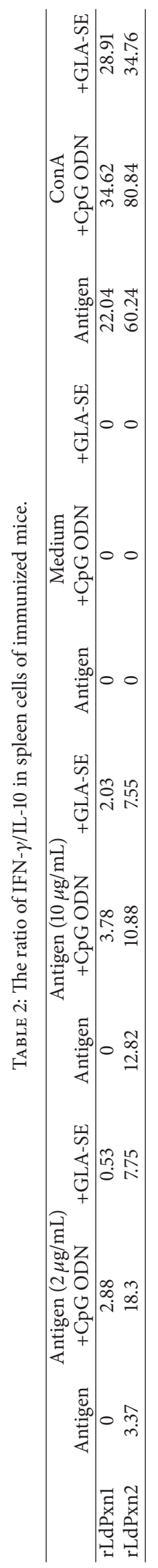




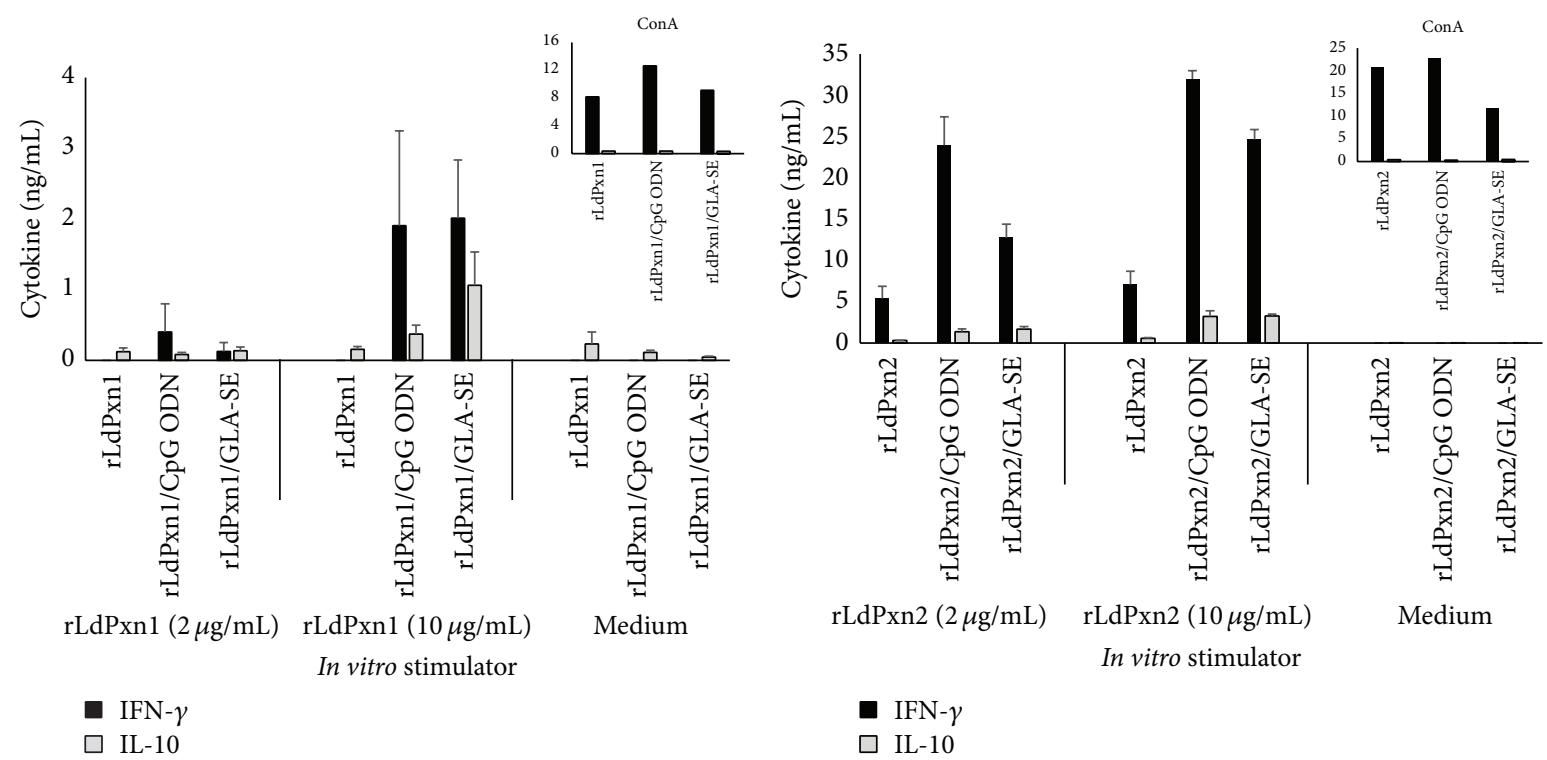

(a)

(b)

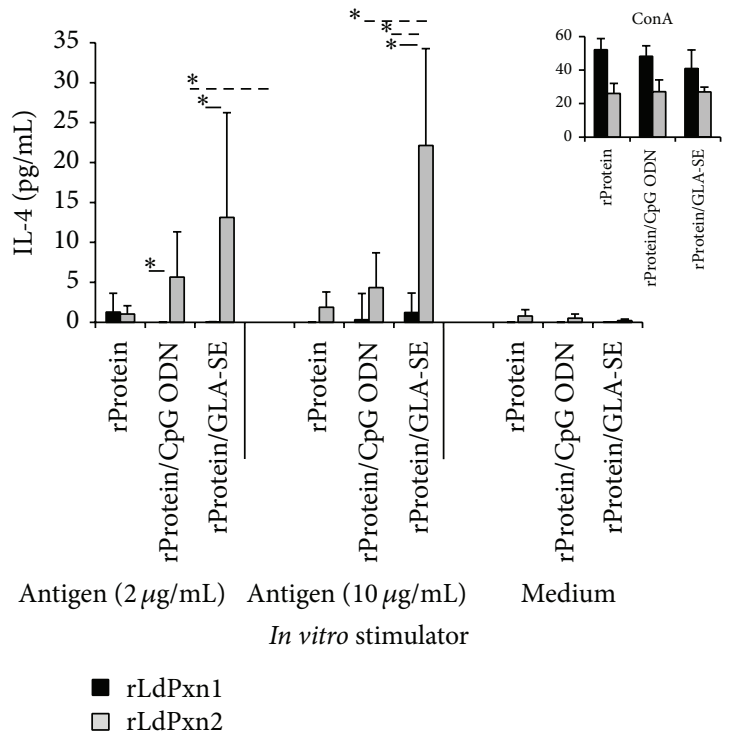

(c)

FIGURE 5: Cytokine responses in spleen cells of rLdPxn1 and rLdPxn2 immunized mice. Mice were immunized s.c. three times at three-week intervals with rLdPxn1 or rLdPxn2 with or without CpG ODN or GLA-SE. Four weeks after the last immunization, spleen cells were prepared and in vitro stimulated with the respective antigen $(2$ and $10 \mu \mathrm{g} / \mathrm{mL})$ or ConA $(5 \mu \mathrm{g} / \mathrm{mL})$. The release of IFN $-\gamma(\mathrm{ng} / \mathrm{mL}), \mathrm{IL}-10(\mathrm{ng} / \mathrm{mL})$, and $\mathrm{IL}-4(\mathrm{pg} / \mathrm{mL})$ in immunized mice was measured in supernatants after $72 \mathrm{hr}$ of in vitro stimulation at $37^{\circ} \mathrm{C}$. Results are presented as the amount of IFN- $\gamma(\mathrm{ng} / \mathrm{mL})$ and IL-10 (ng/mL) for rLdPxn1 (a) and rLdPxn2 (b) or the amount of IL-4 (pg/mL) of rLdPxn1 and rLdPxn2 (c).

unprecedented since several previous studies have also shown the ability of these TLR-based adjuvants to stimulate a protective Thl response against Leishmania antigens [21-27].

The mechanism by which recombinant LdPxnl and LdPxn2 stimulate different immune responses in BALB/c mice was not investigated in this study and it remains to be defined; however, possible explanations are discussed below.

One possibility is that rLdPxn1 and rLdPxn2 are recognized by different TLRs which may result in the stimulation of different effector mechanisms. It has been reported that a mycobacterial early secreted antigenic target protein 6 (ESAT-6) can directly bind to Toll-like receptor 2 and modulate the host immune response [28, 29]. Studies have suggested that lymphocyte-derived cytokines released following TLR ligation can regulate $T$ helper cell differentiation and the type of induced immunity (reviewed in [30]). Additional explanation is that the two antigens may differ in their intracellular trafficking such that they undergo different processing and presentation with major histocompatibility complex (MHC) molecules by antigen presenting cells (APCs). This 
possibility has been proposed as a possible cause for the differences in immune responses triggered against $L$. major TSA and LmsTI1 antigens [31]. Dendritic cells (DCs) are professional antigen presenting cells capable of stimulating T-cell activation [32]. Studies have shown the participation of these cells in Th1/Th2 polarization through differential production of IL-12 and IL-10 [33], as well as IFN- $\gamma$ [34]. Thus, interaction of antigens with DCs is central to the priming and differentiation of T cells.

Also requiring further study is the relationship between rLdPxn1 and rLdPxn2 structures and the immune response as these two antigens may have structural differences, in particular differences in their antigenic epitopes that might affect the humoral immune responses generated against them. Leishmania possesses the typical 2-Cys peroxidoxins which have two conserved cysteine (Cys) residues the peroxidatic cysteine Cys47 located at the $\mathrm{N}$-terminus and the resolving cysteine Cys170 placed near the carboxyl terminus [35, 36]. In general, active peroxidoxins exist as homodimers arranged in a head-to-tail orientation such that the $\mathrm{N}$-terminus cysteine of one monomer is juxtaposed with the C-terminus cysteine on the opposing subunit. The transition of peroxidoxins from the reduced to the oxidized state is commonly associated with a conformational change involving the $\mathrm{C}$-terminus tail. Although the structure of LdPxn1 and LdPxn2 is not available, we anticipate that, following conformational changes (resulting from changes in redox state or from antigen processing), the LdPxn2 C-terminus amino acid extension, composed of the terminal 9 amino acids plus few up-stream amino acids which also exhibit differences from LdPxnl, might present different epitopes and consequently stimulates immune response distinct from LdPxnl. A simple experiment to examine the contribution of LdPxn2 C-terminus extension in shaping the immune response can be done by testing its immunoreactivity in mice. Alternatively, mice immune response to a mutated LdPxn2 molecule depleted of the C-terminus extension or of LdPxn1 molecule to which the LdPxn2 C-terminus extension is introduced can be tested and compared to the immune response against the original molecules.

It is important to note that LdPxn1 and LdPxn2 were examined in the form of recombinant GST-fusion proteins. It has been documented that the immune responses generated against GST-fusion proteins are greatly affected by the carrier portion of the protein as well as the adjuvant used [37, 38]. Moreover, it has been suggested that GST fusion may cause conformational changes of proteins permitted by the flexible linker region [39]. Despite the fact that the rLdPxn1 and rLdPxn2 used in this study were generated in the same way, we believe that it is important to analyze the possible effects that GST fusion may have on the immune response against these proteins.

Our future studies will focus on elucidating the possible mechanisms that regulate mice immune responses against LdPxn1 and LdPxn2. We believe that it is important to understand the potential mechanisms by which these antigens interact with the host immune system to shed light on the factors behind the difference in the immune response to seemingly similar antigens.

\section{Conclusions}

In conclusion, we observed distinct immune response against rLdPxn1 and rLdPxn2 in BALB/c mice. Recombinant LdPxn1 induced a predominant Th2 type whereas rLdPxn2 triggered a mixed $\mathrm{Th} 1 / \mathrm{Th} 2$ with predominant Th1 type response. We also found that CpG ODN and GLA-SE enhance the production of a polarized Thl type regardless of the initial response. In a recent study, we showed that priming with LdPxnl DNA in the presence of murine granulocyte macrophage colonystimulating factor (mGMCSF) and boosting with recombinant LdPxn1 protein stimulates multifunctional $\mathrm{CD} 4^{+} \mathrm{T}$ cells and protects mice against $L$. major infection [40]. It will be interesting to examine the protective effect of recombinant LdPxn1 and LdPxn2 proteins individually or combined with or without adjuvants against Leishmania infection.

\section{Conflict of Interests}

The authors declare that there is no conflict of interests regarding the publication of this paper.

\section{Acknowledgments}

The authors would like to thank Dr. Stephen Barr for cloning LdPxn1 and LdPxn2 in bacterial expression vector. The authors acknowledge Dr. Steve Reed, Infectious Diseases Research Institute, USA, for providing us with GLA-SE adjuvant. The authors also thank Mr. Michael Collier for helping with mice injection. This work was supported by a grant from Canadian Institute of Health Research (CIHR) to Dr. Lashitew Gedamu (FRN 64318).

\section{References}

[1] E. Handman, "Leishmaniasis: current status of vaccine development," Clinical Microbiology Reviews, vol. 14, no. 2, pp. 229-243, 2001.

[2] M. C. De Almeida, V. Vilhena, A. Barral, and M. Barral-Netto, "Leishmanial infection: analysis of its first steps. A review," Memorias do Instituto Oswaldo Cruz, vol. 98, no. 7, pp. 861-870, 2003.

[3] L. Kedzierski and K. J. Evans, "Immune responses during cutaneous and visceral leishmaniasis," Parasitology, vol. 141, no. 12, pp. 1544-1562, 2014.

[4] S. L. Croft, S. Sundar, and A. H. Fairlamb, "Drug resistance in leishmaniasis," Clinical Microbiology Reviews, vol. 19, no. 1, pp. 111-126, 2006.

[5] WHO, Report on the Fifth Consultative Meeting in Leishmania/HIV Coinfection. Addis Ababa, Ethiopia, WHO Document Production Services, Geneva, Swithzerland, 2007.

[6] D. Sacks and C. Anderson, "Re-examination of the immunosuppressive mechanisms mediating non-cure of Leishmania infection in mice," Immunological Reviews, vol. 201, pp. 225238, 2004.

[7] D. Sacks and N. Noben-Trauth, "The immunology of susceptibility and resistance to Leishmania major in mice," Nature Reviews Immunology, vol. 2, no. 11, pp. 845-858, 2002.

[8] H. W. Ghalib, M. R. Piuvezam, Y. A. W. Skeiky et al., "Interleukin 10 production correlates with pathology in human Leishmania 
donovani infections," Journal of Clinical Investigation, vol. 92, no. 1, pp. 324-329, 1993.

[9] F. P. Heinzel, M. D. Sadick, S. S. Mutha, and R. M. Locksley, "Production of Interferon $\gamma$, interleukin 2, interleukin 4, and interleukin 10 by CD4+ lymphocytes in vivo during healing and progressive murine leishmaniasis," Proceedings of the National Academy of Sciences of the United States of America, vol. 88, no. 16, pp. 7011-7015, 1991.

[10] T. L. Stevens, A. Bossie, V. M. Sanders et al., "Regulation of antibody isotype secretion by subsets of antigen-specific helper T cells," Nature, vol. 334, no. 6179, pp. 255-258, 1988.

[11] R. L. Coffman, D. A. Lebman, and P. Rothman, "Mechanism and regulation of immunoglobulin isotype switching," Advances in Immunology, vol. 54, pp. 229-270, 1993.

[12] C. M. Snapper and W. E. Paul, "Interferon- $\gamma$ and B cell stimulatory factor-1 reciprocally regulate Ig isotype production," Science, vol. 236, no. 4804, pp. 944-947, 1987.

[13] J. M. Robinson and J. A. Badwey, "Production of active oxygen species by phagocytic leukocytes," Immunology Series, vol. 60, pp. 159-178, 1994.

[14] S. D. Barr and L. Gedamu, "Role of peroxidoxins in Leishmania chagasi survival. Evidence of an enzymatic defense against nitrosative stress," The Journal of Biological Chemistry, vol. 278, no. 12, pp. 10816-10823, 2003.

[15] R. Chandrashekar, N. Tsuji, T. H. Morales et al., "Removal of hydrogen peroxide by a 1-cysteine peroxiredoxin enzyme of the filarial parasite Dirofilaria immitis," Parasitology Research, vol. 86, no. 3, pp. 200-206, 2000.

[16] M. P. Levick, E. Tetaud, A. H. Fairlamb, and J. M. Blackwell, "Identification and characterisation of a functional peroxidoxin from Leishmania major," Molecular and Biochemical Parasitology, vol. 96, no. 1-2, pp. 125-137, 1998.

[17] S. D. Barr and L. Gedamu, "Cloning and characterization of three differentially expressed peroxidoxin genes from Leishmania chagasi. Evidence for an enzymatic detoxification of hydroxyl radicals," The Journal of Biological Chemistry, vol. 276, no. 36, pp. 34279-34287, 2001.

[18] S. Harder, M. Bente, K. Isermann, and I. Bruchhaus, "Expression of a mitochondrial peroxiredoxin prevents programmed cell death in Leishmania donovani," Eukaryotic Cell, vol. 5, no. 5, pp. 861-870, 2006.

[19] D. B. Smith and K. S. Johnson, "Single-step purification of polypeptides expressed in Escherichia coli as fusions with glutathione S-transferase," Gene, vol. 67, no. 1, pp. 31-40, 1988.

[20] N. S. Daifalla, A. G. Bayih, and L. Gedamu, "Immunogenicity of Leishmania donovani iron superoxide dismutase B1 and peroxidoxin 4 in BALB/c mice: the contribution of Toll-like receptor agonists as adjuvant," Experimental Parasitology, vol. 129, no. 3, pp. 292-298, 2011.

[21] A. Badiee, M. R. Jaafari, A. Samiei, D. Soroush, and A. Khamesipour, "Coencapsulation of CpG oligodeoxynucleotides with recombinant Leishmania major stress-inducible protein 1 in liposome enhances immune response and protection against leishmaniasis in immunized BALB/c mice," Clinical and Vaccine Immunology, vol. 15, no. 4, pp. 668-674, 2008.

[22] S. Bertholet, Y. Goto, L. Carter et al., "Optimized subunit vaccine protects against experimental leishmaniasis," Vaccine, vol. 27, no. 50, pp. 7036-7045, 2009.

[23] S. Iborra, N. Parody, D. R. Abánades et al., "Vaccination with the Leishmania major ribosomal proteins plus CpG oligodeoxynucleotides induces protection against experimental cutaneous leishmaniasis in mice," Microbes and Infection, vol. 10, no. 10-11, pp. 1133-1141, 2008.

[24] M. R. Jaafari, A. Badiee, A. Khamesipour et al., "The role of CpG ODN in enhancement of immune response and protection in $\mathrm{BALB} / \mathrm{c}$ mice immunized with recombinant major surface glycoprotein of Leishmania (rgp63) encapsulated in cationic liposome," Vaccine, vol. 25, no. 32, pp. 6107-6117, 2007.

[25] D. Verthelyi, M. Gursel, R. T. Kenney et al., "CpG oligodeoxynucleotides protect normal and SIV-infected macaques from Leishmania infection," The Journal of Immunology, vol. 170, no. 9, pp. 4717-4723, 2003.

[26] S. Zimmermann, O. Egeter, S. Hausmann et al., "CpG oligodeoxynucleotides trigger protective and curative Th1 responses in lethal murine leishmaniasis," The Journal of Immunology, vol. 160, no. 8, pp. 3627-3630, 1998.

[27] L. Ramírez, D. M. Santos, A. P. Souza et al., "Evaluation of immune responses and analysis of the effect of vaccination of the Leishmania major recombinant ribosomal proteins L3 or L5 in two different murine models of cutaneous leishmaniasis," Vaccine, vol. 31, no. 9, pp. 1312-1319, 2013.

[28] S. Chatterjee, V. P. Dwivedi, Y. Singh et al., "Early secreted antigen ESAT-6 of mycobacterium tuberculosis promotes protective $\mathrm{T}$ helper 17 cell responses in a toll-like receptor-2dependent manner," PLoS Pathogens, vol. 7, no. 11, Article ID e1002378, 2011.

[29] S. K. Pathak, S. Basu, K. K. Basu et al., "Direct extracellular interaction between the early secreted antigen ESAT- 6 of Mycobacterium tuberculosis and TLR2 inhibits TLR signaling in macrophages," Nature Immunology, vol. 8, no. 6, pp. 610-618, 2007.

[30] R. K. Singh, A. Srivastava, and N. Singh, “Toll-like receptor signaling: a perspective to develop vaccine against leishmaniasis," Microbiological Research, vol. 167, no. 8, pp. 445-451, 2012.

[31] R. N. Coler, Y. A. W. Skeiky, K. Bernards et al., "Immunization with a polyprotein vaccine consisting of the T-cell antigens thiol-specific antioxidant, Leishmania major stress-inducible protein 1, and Leishmania elongation initiation factor protects against leishmaniasis," Infection and Immunity, vol. 70, no. 8, pp. 4215-4225, 2002.

[32] E. von Stebut and M. C. Udey, "Requirements for Th1dependent immunity against infection with Leishmania major," Microbes and Infection, vol. 6, no. 12, pp. 1102-1109, 2004.

[33] A. Lanzavecchia and F. Sallusto, "The instructive role of dendritic cells on T cell responses: lineages, plasticity and kinetics," Current Opinion in Immunology, vol. 13, no. 3, pp. 291-298, 2001.

[34] D. Stober, R. Schirmbeck, and J. Reimann, "IL-12/IL-18dependent IFN- $\gamma$ release by murine dendritic cells," The Journal of Immunology, vol. 167, no. 2, pp. 957-965, 2001.

[35] B. Hofmann, H.-J. Hecht, and L. Flohé, "Peroxiredoxins," Biological Chemistry, vol. 383, no. 3-4, pp. 347-364, 2002.

[36] S. McGonigle, J. P. Dalton, and E. R. James, "Peroxidoxins: a new antioxidant family," Parasitology Today, vol. 14, no. 4, pp. 139$145,1998$.

[37] T. M. Daly and C. A. Long, "Influence of adjuvants on protection induced by a recombinant fusion protein against malarial infection," Infection and Immunity, vol. 64, no. 7, pp. 2602-2608, 1996.

[38] C. A. Varley, D. W. Dunne, and J. C. Havercroft, "The influence of adjuvant on humoral responses to glutathione-S-transferase fusion proteins," Parasite Immunology, vol. 14, no. 5, pp. 557$562,1992$. 
[39] D. R. Smyth, M. K. Mrozkiewicz, W. J. McGrath, P. Listwan, and B. Kobe, "Crystal structures of fusion proteins with largeaffinity tags," Protein Science, vol. 12, no. 7, pp. 1313-1322, 2003.

[40] A. G. Bayih, N. S. Daifalla, and L. Gedamu, "DNA-protein immunization using Leishmania peroxidoxin-1 induces a strong $\mathrm{CD}^{+} \mathrm{T}$ cell response and partially protects mice from cutaneous leishmaniasis: role of fusion murine granulocytemacrophage colony-stimulating factor DNA adjuvant," PLoS Neglected Tropical Diseases, vol. 8, no. 12, article e3391, 2014. 


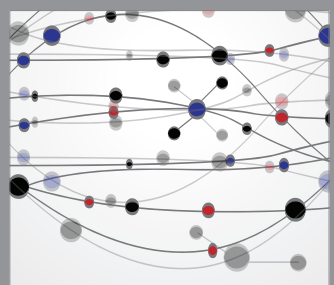

The Scientific World Journal
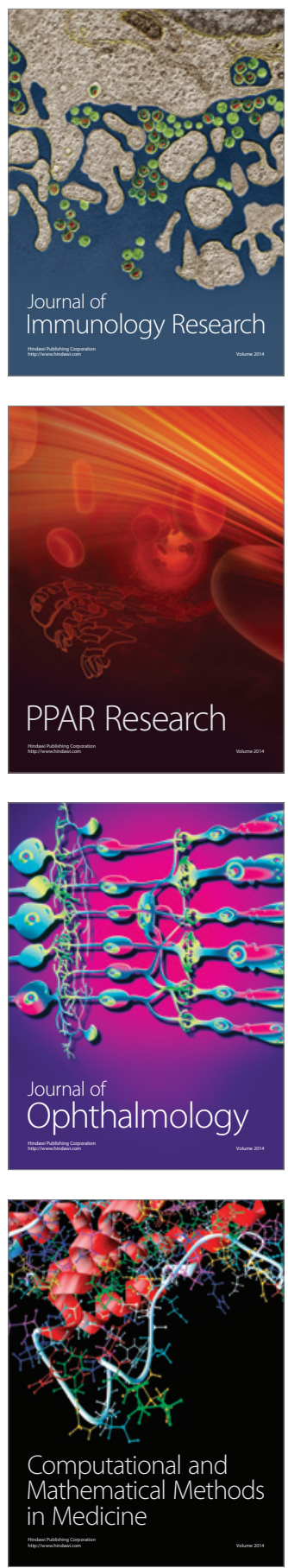

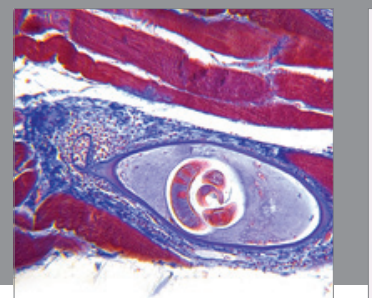

Gastroenterology

Research and Practice
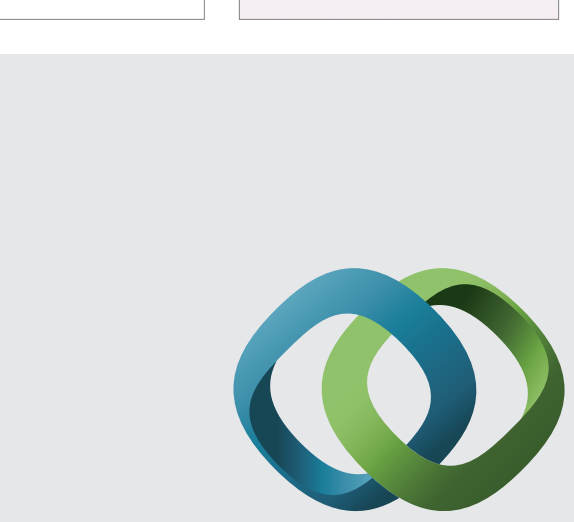

\section{Hindawi}

Submit your manuscripts at

http://www.hindawi.com
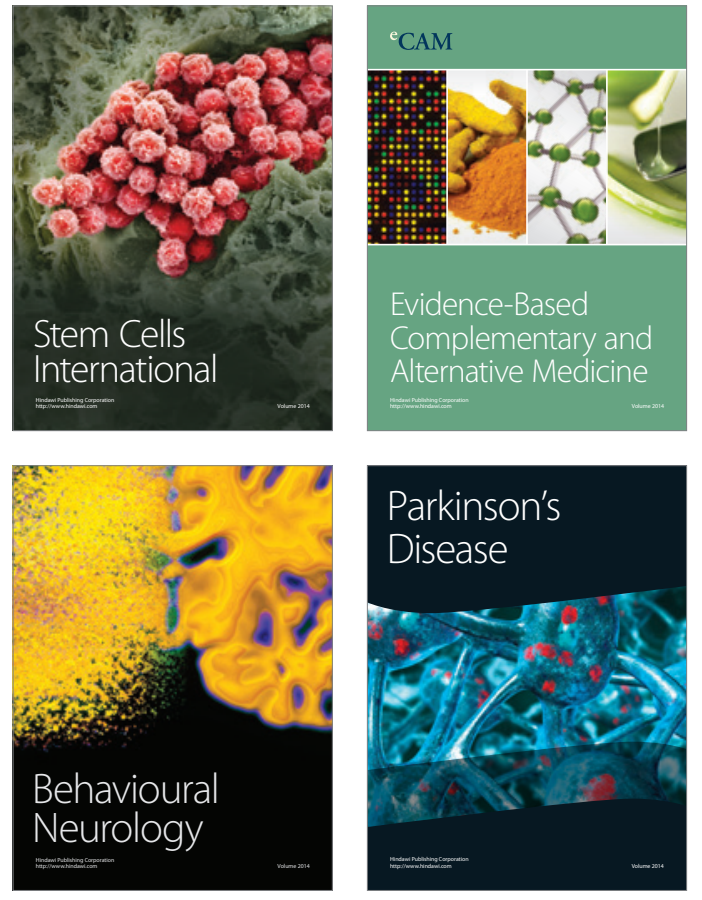
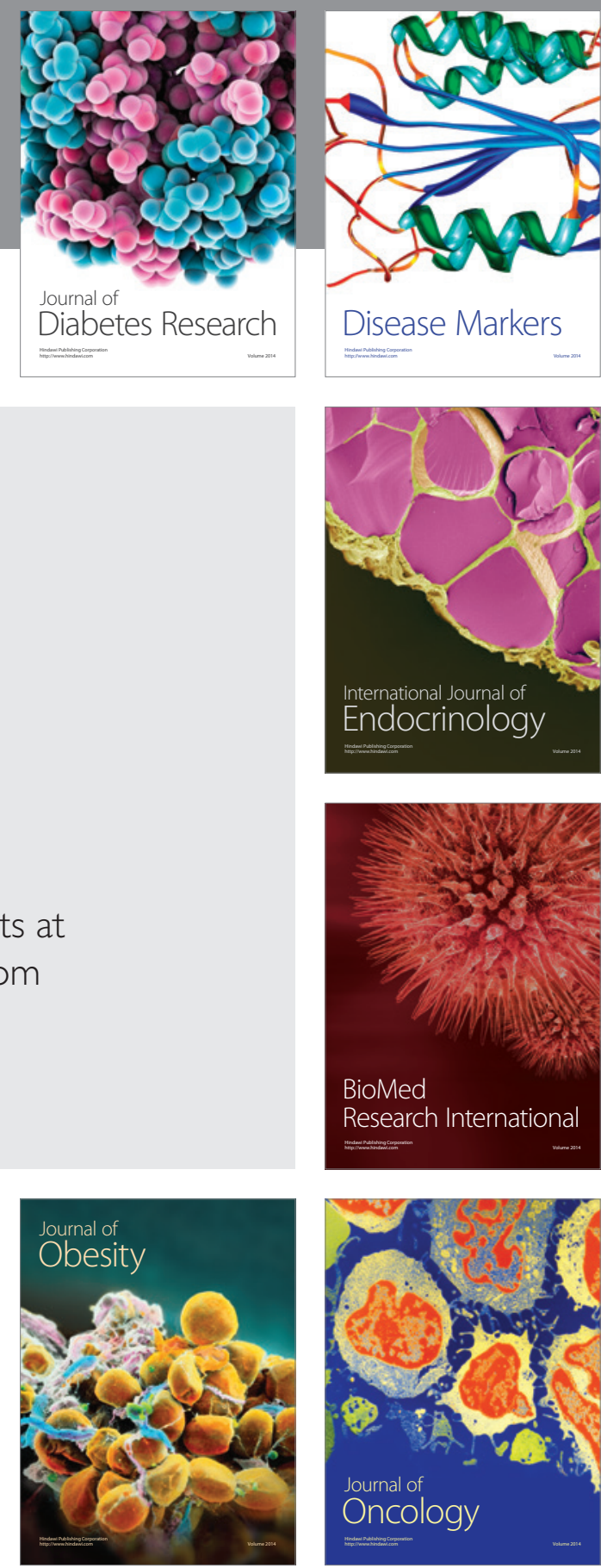

Disease Markers
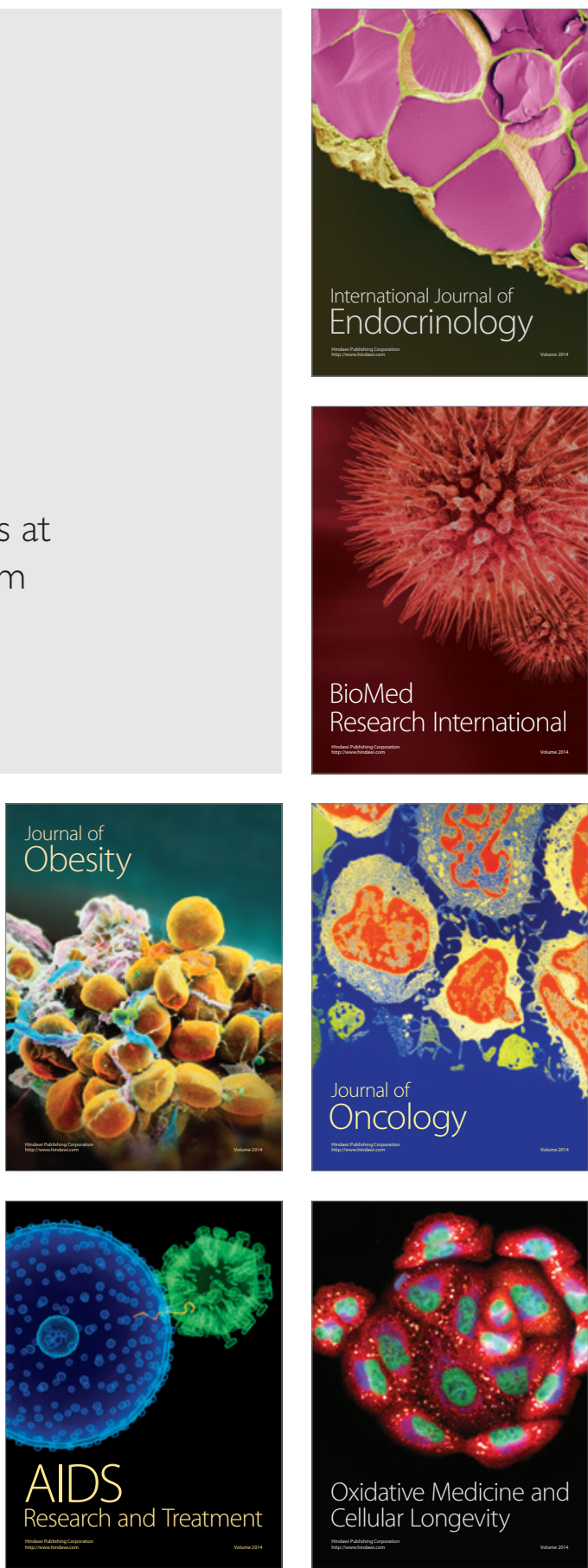